\title{
Prevalence and Factors Associated With Dental Fluorosis among Female School Going Children Aged 12-15 Years in Southern Sub- Population of Saudi Arabia
}

\author{
Eroje Alezi Braimoh Ifindon (BDS, MDPH) ${ }^{1 *}$, Tikare Shreyas (BDS, MDS) ${ }^{2}$, Khaleelahmed Shaeesta B (BDS, MDS) ${ }^{3}$, \\ Udeabor Samuel Ebele (BDS, FWACS) ${ }^{4}$, Al-wadai Batool Mohammed (BDS) ${ }^{5}$, Al-beshi Shahad Moshhen (BDS) ${ }^{6}$ \\ ${ }^{1}$ Assistant Professor, Department of Periodontics and Community Dental Sciences, King Khalid University, Abha, Kingdom of Saudi Arabia \\ ${ }^{2}$ Assistant Professor, Department of Periodontics and Community Dental Sciences, King Khalid University, Abha, Kingdom of Saudi Arabia \\ ${ }^{3}$ Assistant Professor, Department of Periodontics and Community Dental Sciences, King Khalid University, Abha, Kingdom of Saudi Arabia \\ ${ }^{4}$ Assistant Professor, Department of Oral and Maxillofacial Surgery, Abha, Kingdom of Saudi Arabia \\ ${ }^{5}$ Intern Dentist, Department of Periodontics and Community Dental Sciences, King Khalid University, Abha, Kingdom of Saudi Arabia \\ ${ }^{6}$ Intern Dentist, Department of Periodontics and Community Dental Sciences, King Khalid University, Abha, Kingdom of Saudi Arabia
}

DOI: 10.36348/SJODR.2019.v04i09.009

| Received: 08.09.2019 | Accepted: 14.09.2019| Published: 29.09.2019

*Corresponding author: Eroje Alezi Braimoh Ifindon

Abstract

Objectives: This study aims to determine the prevalence of dental fluorosis and the factors associated with dental fluorosis among 12-15 years old female school children in southern sub-population of Saudi Arabia. Methodology: A community-based, cross-sectional survey of 486 school children (females), 12-15 years age group in southern subpopulation of Saudi Arabia using. Clinical examinations were performed using the Dean's index by WHO 2013. A pretested questionnaire was used to gather exploratory data on Demographics, Socioeconomic conditions, Access to dental service and exposure to various sources of fluoride. Bivariate associations were examined using the Chi-square and Chisquare trend tests to evaluate the association of selected risk factors with the presence or absence of dental fluorosis. Samples of water were collected from water sources consumed and analyzed. Results: The overall prevalence of dental fluorosis in our study sample was $59.05 \%$ and the Community Fluorosis Index (CFI) was 0.4. Questionable fluorosis $(50.21 \%)$ was the commonest fluorosis seen. The principal factor associated with the presence of dental fluorosis was the frequency of tooth brushing and the use of fluoride supplements with a p-value of 0.04060 and 0.0497 respectively. No significant association was seen between dental fluorosis and socioeconomic status or parental education, drinking water and the intake of tea/coffee, consumption of any type of fish. Conclusions: Dental fluorosis is a major public health issue in the southern sub-population of Saudi Arabia. This study showed borderline public health significance with a CFI of 0.4. The principal factor associated with the presence of dental fluorosis in this tested model was the frequency of tooth brushing and the use of fluoride supplements. Active steps/strategies must be taken to educate the community on the correct frequency of tooth brushing during childhood development and the medical practitioners on the correct prescription of fluorides supplements during the stages of tooth development.

Keywords: Dental; fluoridation; risk factors; water fluoride level; Saudi Arabia.

Copyright @ 2019: This is an open-access article distributed under the terms of the Creative Commons Attribution license which permits unrestricted use, distribution, and reproduction in any medium for non-commercial use (NonCommercial, or CC-BY-NC) provided the original author and source are credited.

\section{INTRODUCTION}

Fluoride is an essential element for human health and it plays a critical role in the calcification of bones and teeth [1]. Fluoride is often called a doubleedged sword because fluoride intake deficiency leads to dental caries while excessive exposure to fluoride leads to dental fluorosis, skeletal fluorosis and non-vertebral fractures especially hip fractures [2].

Dental fluorosis is a specific disturbance of tooth enamel formation characterized by the greater surface and sub-surface porosity that may attract extrinsic stains, resulting to discolouration produced by chronic ingestion of excessive amount of fluoride [3]. Dental fluorosis is one of the common but major emerging areas of research in the tropics and is considered a major public health issue [4].

In the Kingdom of Saudi Arabia, certain populations also suffer from the menace of dental fluorosis as a result of abundance of well water still being consumed in the remote parts of the country [5] as well as increased fluorosis experienced among 
children residing in high altitudes areas [6]. In some rural areas of Saudi Arabia where drinking water is obtained from wells about $300 \mathrm{~m}$ deep, the prevalence of dental fluorosis is as high as $90 \%$. Fluoride content of these wells ranges between $0.5 \mathrm{ppm}-2.5 \mathrm{ppm}$ [3].

The principal source of fluoride is drinking water and the optimal cario-protective fluoride content in drinking water is approximately $1 \mathrm{ppm}$ in temperate climates while in tropical climates where people drink more water due to hot climate, desirable fluoride content of drinking water may be $0.5 \mathrm{ppm}$ [1]. However, professionally and self-applied tropical fluorides products, dietary fluorides supplements, toothpaste with fluoride, beverages and food prepared with fluoridated drinking water can be other sources of exposure [2]. The greatest risk factors associated with dental fluorosis is the total amount of fluoride consumed from all sources during the critical period of tooth development [7].

Dental fluorosis affects dental enamel structure by altering its shape and results in aesthetic and functional problems depending on the severity of the lesions. Poor aesthetics due to fluorosis may have psychological, financial and behavioural implications for the quality of life of the individual [8].

However, despite the substantial research studies in the western context on fluorosis there is still a definite gap in the literature in the Middle East especially on the possible risk factors associated with dental fluorosis [5]. To the best of our knowledge and the available literature in Saudi Arabia particularly in the southern sub-population (Abha-Khamis) region, where this research is carried out there is insufficient studies to reach any conclusion about dental fluorosis and its associated factors [5].

It is therefore imperative to conduct a quantitative cross-sectional study using a probability purposive sampling methodology in the selected female schools in Abha-Khamis to establish the statistical relationship between dental fluorosis and the associated risk factors. The findings of this research might be of great benefits to the entire community and the country at large by establishing a suitable policy to ensure effective preventive management strategy. Objectives of the study:

- To determine the prevalence of dental fluorosis among the school children in the study population

- To determine the factors associated with dental fluorosis among female school going children aged 12-15 years old in southern subpopulation of Saudi Arabia.

\section{MATERIALS AND METHODS}

This present study was approved by the King Khalid University, College of Dentistry (KKU-COD) scientific research committee (SRC) with registration number SRC/2017-2018/92, All procedures followed in this study were at par with the international standards for best practices recommended by the World Medical Association (WMA) declaration of Helsinki in conducting with human research.

\section{Study Location}

This study was carried out in Abha-Khamis, the capital of Aseer province of Saudi Arabia. Abha is located in the South- western region of Saudi Arabia with a population of over 600,000 people [9]. It is situated 2,200 meters above the sea level in the fertile mountains of South- western part of the kingdom of Saudi Arabia, with a mild cold climate and semi- arid in nature making it popular tourist destination/attraction [10].

\section{Sample Selection}

Children in these age groups were selected because they represent a population at risk for dental fluorosis: the period of calcification of teeth from infancy to 6 years of age constitutes the vulnerable period for the onset of the condition.

We conducted a community-based, crosssectional survey of 486 school children (females) in the age group of 12-1 5 years living in the city of Southern sub-population of Saudi Arabia, using a simple random sampling technique. In consultation with the Ministry of Education, we compiled a list of various schools which had grade 6 to grade 9 where these age groups are found. List of 168 public and private schools both in Abha and Khamis were obtained from the ministry of education and from the list 40 schools (20 each from Abha and Khamis) were randomly selected using random number tables, expecting to get more than 350 children on the basis of the average class strength using population size. All students selected in each school were included in the study. Prior to the study information was given to the school directors and students about the date and time of data collection, a written informed consent was obtained from various schools involved and a verbal consent from the children to participate in the study was equally taken.

\section{Questionnaire}

The data were collected and recorded, based on the a structured closed-opened pre-tested questionnaire to obtained information on socioeconomic status, occupation and educational level of parents, sources of drinking, tea/coffee consumption, sea-fish intake and use of fluoride- containing toothpaste by the students. These factors have been identified as associated risk factors for dental fluorosis in previous studies [8]. Printed questionnaire was translated into Arabic language and directly administered to the students with the help of the trained dental investigators/specialist. 


\section{Clinical Examination}

Clinical examination was carried out using dental mirrors and probes, the investigators were calibrated and dental fluorosis classification using modified Dean's index 2013 by WHO were performed prior to the commencement of the study in 10 schools from both Abha and Khamis axis. A pilot study of two examiners and 20 students revealed high intra-and interexaminer reproducibility of the use of Dean's index (Kappa 0.90). Data were collected in December 2018 under natural and artificial light after careful drying of the teeth with cotton wools. Written informed consent was obtained from the various schools directors and verbal consent from the children to participate in the study was taken.

The prevalence and severity of dental fluorosis was assessed using modified Dean's index by WHO recommendations [11] and Community Fluorosis Index (CFI). The modified Dean's index by WHO 2013 was used to determine the grades of dental fluorosis as follows:

$0=$ Normal-white pale creamy, glossy and smooth surface enamel

1=Questionable-slight aberrations ranges from few white flecks to occasional spots mainly on the incisor tips and cusp tips.

$2=$ Very mild-small, opaque, paper-white areas scattered irregularly involving less than $25 \%$

$3=$ Mild-white opacities of the enamel involving more than $25 \%$ but less than $50 \%$

4=Moderate-show marked wear, and brown staining is frequently disfiguring feature

$5=$ Severe-enamel severely affected and the hypoplasia is so marked with pitting or worn areas and brown stains with corroded appearance.

$8=$ Excluded crown, restorations, brackets

$9=$ Not recorded (unerupted tooth)

The prevalence of dental fluorosis was estimated by taking all cases of dental fluorosis as the numerator and the total child population seen in the age groups as the denominator.

A Community Fluorosis Index (CFI) was calculated by summating the scores of individual grade with weighed scores (as described by Dean) and dividing the sum by the total sample size. A CFI of greater than 0.4 has been used to identify areas where fluorosis is a public health concern [1].

The public health significance of CFI values is shown below:

\begin{tabular}{|l|l|}
\hline CFI value range & Public health significance \\
\hline $0.0-4$ & Negative \\
\hline $0.4-0.6$ & Borderline \\
\hline $0.6-1.0$ & Slight \\
\hline $1.0-2.0$ & Medium \\
\hline $2.0-3.0$ & Marked \\
\hline $3.0-4.0$ & Very marked \\
\hline
\end{tabular}

All analyses were performed using SPSS version 20.0 and a $p$ value of $<0.05$ was taken to indicate statistical significance level.

\section{Water Fluoride Content}

Samples of water were collected from water sources consumed by the respondents in each location and the water fluoride content in the study area was obtained and sent to the Department of Chemistry, King Khalid University for analysis and an average of $0.7 \mathrm{ppm}$ fluoride content was obtained.

\section{Statistical Analysis}

Assuming a margin of error at 5\% and confidence interval of $95 \%$ using an estimated population size of 500,000 , the estimated sample size for this study was calculated to be 384 . We conducted a community-based, cross-sectional survey of 486 school children (females) in the age group of 12-1 5 years in Southern sub-population of Saudi Arabia. The independent variables were categorized into the following; Demographic data, socioeconomic status, access to dental services and oral hygiene habits. The dependent variable dental fluorosis was categorized according to the degree of severity of dental fluorosis described above. Data were stored and imported to SPSS 20.0 software for statistical analysis. After exploratory analysis, descriptive statistics, inferential statistical testing was performed to examine the association between the outcome variable and other independent variables using a bivariate analysis with Chi-square tests and Chi-square trend tests.

\section{Inclusion Criteria}

- Being a resident in the city of Abha-Khamis since birth or moving to the city before the age of 2 years

- Absence of fixed Orthodontics appliances

- Signature of an informed consent form by parents or guardian responsible for the children

- All 12-15 years old children present on the day of clinical examination

- Children with permanent teeth and no fillings on facial surfaces

\section{Exclusion Criteria}

- Children with other fluorosis stains are excluded from the study

- Children with Orthodontics brackets or crowns are not included

- Children who had migrated from some other place or who were not permanent residents of the study area

- All children who are celebrating their $16^{\text {th }}$ birthday and above on the day of clinical examination are excluded. 


\section{RESULTS}

The present study comprised of 486 subjects, out of which $57 \%$ reside in urban (city) area and $43 \%$ in town. Subjects belonging to Saudi and non-Saudi ethic group were $95.06 \%$ and $4.94 \%$ respectively. School grade $6,7,8$ and 9 was reported in $58.44 \%, 17.49 \%$, $14.61 \%$ and $9.47 \%$ subjects respectively. The subjects $(63.99 \%, 17.49 \%, 11.93 \%$ and $6.58 \%)$ belonged to 12 , 13,14 and 15 year age respectively. Subjects belonging to public and private schools were $64.40 \%$ and $35.60 \%$ respectively (Table-1).

Table-1: Distribution of respondents by different demographic characteristics

\begin{tabular}{|l|c|c|}
\hline Factors & No of respondents & \% of respondents \\
\hline Location type & 277 & 57.00 \\
\hline Urban & 209 & 43.00 \\
\hline Town & \multicolumn{2}{|l|}{} \\
\hline Ethnic groups & 462 & 95.06 \\
\hline Saudi & 24 & 4.94 \\
\hline Non-Saudi & 284 & 58.44 \\
\hline Years in school & 85 & 17.49 \\
\hline Grade 6 & 71 & 14.61 \\
\hline Grade 7 & 46 & 9.47 \\
\hline Grade 8 & 311 & 63.99 \\
\hline Grade 9 & 85 & 17.49 \\
\hline Age in years & 58 & 11.93 \\
\hline 12 years & 32 & 6.58 \\
\hline 13 years & 313 & 64.40 \\
\hline 14 years & 173 & 35.60 \\
\hline 15 years & 486 & 100.00 \\
\hline Types of school &
\end{tabular}

Normal, questionable, very mild and mild enamel fluorosis were revealed in $40.95 \%, 50.21 \%$, $6.17 \%$ and $2.67 \%$ of the subjects respectively (table 2 , graph 1). Normal fluorosis was more in urban (43.6\%) than rural population $(37.3 \%)$. Mild fluorosis was reported in $3.25 \%$ and $1.91 \%$ of the urban and rural subjects respectively. When different categories of fluorosis was compared statistically among urban and rural population, it was found to be statistically significant as $\mathrm{p}<0.05$. Non-Saudi and Saudi ethnic group had 29.17 and $51.3 \%$ questionable fluorosis respectively while mild fluorosis was revealed in $4.17 \%$ and $2.60 \%$ of the subjects respectively with statistically insignificant difference. Questionable fluorosis was found highest in grade 9 whereas mild and very mild in grade 6 school subjects with statistically insignificant difference. Subjects aged 12, 13, 14 and 15 years when compared statistically according to different categories of fluorosis, were found to be statistically significant (Table-3).

Table-2: Prevalence of enamel fluorosis

\begin{tabular}{|l|c|c|}
\hline Enamel fluorosis & $\begin{array}{c}\text { No of } \\
\text { respondents }\end{array}$ & $\begin{array}{c}\text { \% of } \\
\text { respondents }\end{array}$ \\
\hline Normal & 199 & 40.95 \\
\hline Questionable & 244 & 50.21 \\
\hline Very mild & 30 & 6.17 \\
\hline Mild & 13 & 2.67 \\
\hline Total & 486 & 100.00 \\
\hline
\end{tabular}

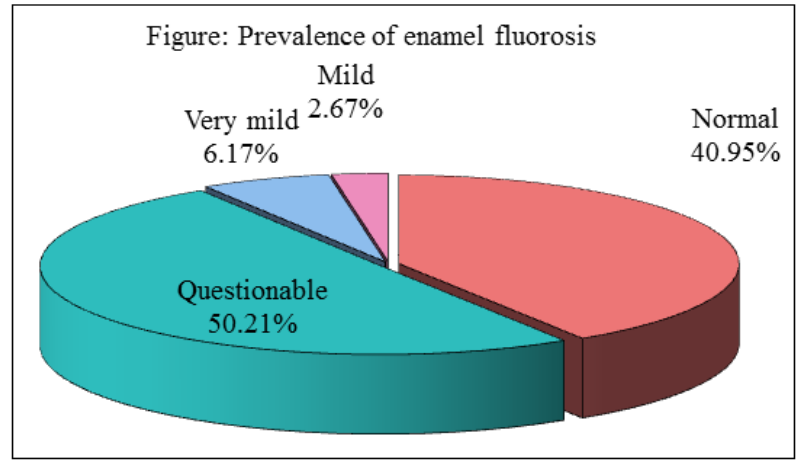

Fig-1: Prevalence of enamel fluorosis

Table-3: Association between demographic characteristics with prevalence of enamel fluorosis

\begin{tabular}{|c|c|c|c|c|c|c|c|c|c|c|c|c|}
\hline Factors & $\begin{array}{c}\text { Norma } \\
1\end{array}$ & $\%$ & $\begin{array}{c}\text { Questionabl } \\
\text { e }\end{array}$ & $\%$ & $\begin{array}{l}\text { Very } \\
\text { mild }\end{array}$ & $\%$ & $\begin{array}{c}\text { Mil } \\
\text { d }\end{array}$ & $\%$ & $\begin{array}{c}\text { Tota } \\
1\end{array}$ & $\%$ & $\begin{array}{c}\text { Chi- } \\
\text { square }\end{array}$ & $\begin{array}{c}\text { p- } \\
\text { value }\end{array}$ \\
\hline \multicolumn{13}{|l|}{$\begin{array}{l}\text { Location } \\
\text { type }\end{array}$} \\
\hline Urban & 121 & $\begin{array}{c}43.6 \\
8\end{array}$ & 125 & $\begin{array}{c}45.1 \\
3\end{array}$ & 22 & $\begin{array}{c}7.9 \\
4\end{array}$ & 9 & $\begin{array}{c}3.2 \\
5\end{array}$ & 277 & 57.00 & 8.5484 & $\begin{array}{c}0.0360 \\
*\end{array}$ \\
\hline Town & 78 & $\begin{array}{c}37.3 \\
2 \\
\end{array}$ & 119 & $\begin{array}{c}56.9 \\
4 \\
\end{array}$ & 8 & $\begin{array}{c}3.8 \\
3 \\
\end{array}$ & 4 & $\begin{array}{c}1.9 \\
1 \\
\end{array}$ & 209 & 43.00 & & \\
\hline \multicolumn{13}{|l|}{$\begin{array}{l}\text { Ethnic } \\
\text { groups }\end{array}$} \\
\hline Saudi & 185 & $\begin{array}{c}40.0 \\
4\end{array}$ & 237 & $\begin{array}{c}51.3 \\
0\end{array}$ & 28 & $\begin{array}{c}6.0 \\
6\end{array}$ & 12 & $\begin{array}{c}2.6 \\
0\end{array}$ & 462 & 95.06 & 4.4908 & 0.2131 \\
\hline Non-Saudi & 14 & $\begin{array}{c}58.3 \\
3 \\
\end{array}$ & 7 & $\begin{array}{c}29.1 \\
7\end{array}$ & 2 & $\begin{array}{c}8.3 \\
3 \\
\end{array}$ & 1 & $\begin{array}{c}4.1 \\
7\end{array}$ & 24 & 4.94 & & \\
\hline \multicolumn{13}{|l|}{$\begin{array}{l}\text { Years in } \\
\text { school }\end{array}$} \\
\hline Grade 6 & 118 & $\begin{array}{c}41.5 \\
5\end{array}$ & 134 & $\begin{array}{c}47.1 \\
8\end{array}$ & 23 & $\begin{array}{c}8.1 \\
0\end{array}$ & 9 & $\begin{array}{c}3.1 \\
7\end{array}$ & 284 & 58.44 & 8.2833 & 0.5059 \\
\hline Grade 7 & 34 & $\begin{array}{c}40.0 \\
0\end{array}$ & 45 & $\begin{array}{c}52.9 \\
4\end{array}$ & 5 & $\begin{array}{c}5.8 \\
8\end{array}$ & 1 & $\begin{array}{c}1.1 \\
8\end{array}$ & 85 & 17.49 & & \\
\hline
\end{tabular}


Eroje Alezi Braimoh Ifindon et al; Saudi J Oral Dent Res, Sep 2019; 4(9): 612-622

\begin{tabular}{|c|c|c|c|c|c|c|c|c|c|c|c|c|}
\hline Grade 8 & 28 & $\begin{array}{c}39.4 \\
4\end{array}$ & 39 & $\begin{array}{c}54.9 \\
3\end{array}$ & 2 & $\begin{array}{c}2.8 \\
2\end{array}$ & 2 & $\begin{array}{c}2.8 \\
2\end{array}$ & 71 & 14.61 & & \\
\hline Grade 9 & 19 & $\begin{array}{c}41.3 \\
0\end{array}$ & 26 & $\begin{array}{c}56.5 \\
2\end{array}$ & 0 & $\begin{array}{c}0.0 \\
0\end{array}$ & 1 & $\begin{array}{c}2.1 \\
7\end{array}$ & 46 & 9.47 & & \\
\hline \multicolumn{13}{|l|}{$\begin{array}{l}\text { Age in } \\
\text { years }\end{array}$} \\
\hline 12 years & 131 & $\begin{array}{c}42.1 \\
2\end{array}$ & 147 & $\begin{array}{c}47.2 \\
7\end{array}$ & 24 & $\begin{array}{c}7.7 \\
2 \\
\end{array}$ & 9 & $\begin{array}{c}2.8 \\
9 \\
\end{array}$ & 311 & 63.99 & 16.9885 & $\begin{array}{c}0.0489 \\
* \\
\end{array}$ \\
\hline 13 years & 34 & $\begin{array}{c}40.0 \\
0\end{array}$ & 44 & $\begin{array}{c}51.7 \\
6\end{array}$ & 6 & $\begin{array}{c}7.0 \\
6\end{array}$ & 1 & $\begin{array}{c}1.1 \\
8\end{array}$ & 85 & 17.49 & & \\
\hline 14 years & 23 & $\begin{array}{c}39.6 \\
6\end{array}$ & 34 & $\begin{array}{c}58.6 \\
2\end{array}$ & 0 & $\begin{array}{c}0.0 \\
0\end{array}$ & 1 & $\begin{array}{c}1.7 \\
2\end{array}$ & 58 & 11.93 & & \\
\hline 15 years & 11 & $\begin{array}{c}34.3 \\
8\end{array}$ & 19 & $\begin{array}{c}59.3 \\
8\end{array}$ & 0 & $\begin{array}{c}0.0 \\
0\end{array}$ & 2 & $\begin{array}{c}6.2 \\
5\end{array}$ & 32 & 6.58 & & \\
\hline \multicolumn{13}{|l|}{$\begin{array}{l}\text { Types of } \\
\text { school }\end{array}$} \\
\hline $\begin{array}{l}\text { Public } \\
\text { school }\end{array}$ & 139 & $\begin{array}{c}44.4 \\
1\end{array}$ & 144 & $\begin{array}{c}46.0 \\
1\end{array}$ & 19 & $\begin{array}{c}6.0 \\
7\end{array}$ & 11 & $\begin{array}{c}3.5 \\
1\end{array}$ & 313 & 64.40 & 7.9945 & $\begin{array}{c}0.0461 \\
*\end{array}$ \\
\hline $\begin{array}{l}\text { Private } \\
\text { school }\end{array}$ & 60 & $\begin{array}{c}34.6 \\
8\end{array}$ & 100 & $\begin{array}{c}57.8 \\
0\end{array}$ & 11 & $\begin{array}{c}6.3 \\
6\end{array}$ & 2 & $\begin{array}{c}1.1 \\
6\end{array}$ & 173 & 35.60 & & \\
\hline Total & 199 & $\begin{array}{c}40.9 \\
5\end{array}$ & 244 & $\begin{array}{c}50.2 \\
1\end{array}$ & 30 & $\begin{array}{c}6.1 \\
7\end{array}$ & 13 & $\begin{array}{c}2.6 \\
7\end{array}$ & 486 & $\begin{array}{c}100.0 \\
0\end{array}$ & & \\
\hline
\end{tabular}

The mothers $(51.65 \%)$ work outside the home. More than half $(55.97 \%)$ of the subjects mother had studied up-to university level. Most common source of drinking water was bottled water $(62.96 \%)$ followed by both tap/bottled/well water $(33.74 \%)$. Dry fish and tea was consumed by $10.70 \%$ and $3.91 \%$ respectively (Table-4).

Table-4: Economic status related factor wise distribution of respondents

\begin{tabular}{|c|c|c|}
\hline Factors & No of respondents & $\%$ of respondents \\
\hline \multicolumn{3}{|c|}{ What is your parent monthly family income } \\
\hline 3,000SR- 4,000SR & 27 & 5.56 \\
\hline 3,000SR- 4,000SR & 459 & 94.44 \\
\hline \multicolumn{3}{|c|}{ What is your mother's occupation } \\
\hline Full housewife & 235 & 48.35 \\
\hline Working out of the home & 251 & 51.65 \\
\hline \multicolumn{3}{|c|}{ What is your mother's educational level } \\
\hline High school & 182 & 37.45 \\
\hline Vocational & 25 & 5.14 \\
\hline University & 272 & 55.97 \\
\hline Non-educated & 7 & 1.44 \\
\hline \multicolumn{3}{|c|}{ What is your source of drinking water } \\
\hline Bottled water & 306 & 62.96 \\
\hline Well water & 16 & 3.29 \\
\hline Tap/Public water & 0 & 0.00 \\
\hline Both tap/bottled/well water & 164 & 33.74 \\
\hline \multicolumn{3}{|c|}{ What is the type of tea or fish do you take } \\
\hline None & 26 & 5.35 \\
\hline Tea & 19 & 3.91 \\
\hline Coffee & 14 & 2.88 \\
\hline Both tea/coffee & 15 & 3.09 \\
\hline Sea-fish (fresh) & 29 & 5.97 \\
\hline Dry-fish & 52 & 10.70 \\
\hline Others & 331 & 68.11 \\
\hline Total & 486 & 100.00 \\
\hline
\end{tabular}

Very mild and mild fluorosis was reported most in subjects whose mothers were illiterate and studied up-to vocational level respectively. When parents monthly income, mother's occupation, mother's educational level, source of drinking water, the type of tea and fish consumption were compared according to the categories of fluorosis, it was found to be statistically insignificant (Table-5). 
Eroje Alezi Braimoh Ifindon et al; Saudi J Oral Dent Res, Sep 2019; 4(9): 612-622

Table-5: Association between socio economic status related factors with prevalence of enamel fluorosis

\begin{tabular}{|c|c|c|c|c|c|c|c|c|c|c|c|c|}
\hline Factors & $\begin{array}{c}\text { Norm } \\
\text { al }\end{array}$ & $\%$ & $\begin{array}{c}\text { Questiona } \\
\text { ble }\end{array}$ & $\%$ & $\begin{array}{l}\text { Very } \\
\text { mild }\end{array}$ & $\%$ & $\begin{array}{c}\text { Mil } \\
\mathrm{d}\end{array}$ & $\%$ & $\begin{array}{c}\text { Tot } \\
\text { al }\end{array}$ & $\%$ & $\begin{array}{c}\text { Chi- } \\
\text { square }\end{array}$ & $\begin{array}{c}\mathrm{p}- \\
\text { value }\end{array}$ \\
\hline \multicolumn{13}{|c|}{ What is your parent monthly family income? } \\
\hline 3,000SR- 4,000SR & 8 & $\begin{array}{l}29 . \\
63\end{array}$ & 14 & $\begin{array}{l}51 . \\
85\end{array}$ & 3 & 11. & 2 & $\begin{array}{c}7.4 \\
1\end{array}$ & 27 & 5.56 & 4.4327 & $\begin{array}{c}0.218 \\
4\end{array}$ \\
\hline 3,000SR- 4,000SR & 191 & $\begin{array}{l}41 . \\
61\end{array}$ & 230 & $\begin{array}{c}50 . \\
11 \\
\end{array}$ & 27 & $\begin{array}{c}5.8 \\
8\end{array}$ & 11 & $\begin{array}{c}2.4 \\
0\end{array}$ & 459 & $\begin{array}{c}94.4 \\
4\end{array}$ & & \\
\hline \multicolumn{13}{|c|}{ What is your mother's occupation? } \\
\hline Full housewife & 90 & $\begin{array}{l}38 . \\
30\end{array}$ & 123 & $\begin{array}{l}52 . \\
34\end{array}$ & 17 & $\begin{array}{c}7.2 \\
3\end{array}$ & 5 & $\begin{array}{c}2.1 \\
3\end{array}$ & 235 & $\begin{array}{c}48.3 \\
5\end{array}$ & 2.5321 & $\begin{array}{c}0.469 \\
5\end{array}$ \\
\hline $\begin{array}{l}\text { Working out of the } \\
\text { home }\end{array}$ & 109 & $\begin{array}{l}43 . \\
43\end{array}$ & 121 & $\begin{array}{l}48 . \\
21\end{array}$ & 13 & $\begin{array}{c}5.1 \\
8\end{array}$ & 8 & $\begin{array}{c}3.1 \\
9\end{array}$ & 251 & $\begin{array}{c}51.6 \\
5\end{array}$ & & \\
\hline \multicolumn{13}{|c|}{ What is your mother's educational level? } \\
\hline High school & 70 & $\begin{array}{l}38 . \\
46\end{array}$ & 97 & $\begin{array}{l}53 . \\
30\end{array}$ & 11 & $\begin{array}{c}6.0 \\
4\end{array}$ & 4 & $\begin{array}{c}2.2 \\
0\end{array}$ & 182 & $\begin{array}{c}37.4 \\
5\end{array}$ & 6.0292 & $\begin{array}{c}0.737 \\
0\end{array}$ \\
\hline Vocational & 8 & $\begin{array}{l}32 . \\
00\end{array}$ & 16 & $\begin{array}{l}64 . \\
00\end{array}$ & 0 & $\begin{array}{c}0.0 \\
0\end{array}$ & 1 & $\begin{array}{c}4.0 \\
0\end{array}$ & 25 & 5.14 & & \\
\hline University & 118 & $\begin{array}{l}43 . \\
38\end{array}$ & 128 & $\begin{array}{l}47 . \\
06\end{array}$ & 18 & $\begin{array}{c}6.6 \\
2\end{array}$ & 8 & $\begin{array}{c}2.9 \\
4\end{array}$ & 272 & $\begin{array}{c}55.9 \\
7\end{array}$ & & \\
\hline Non-educated & 3 & $\begin{array}{l}42 . \\
86\end{array}$ & 3 & $\begin{array}{l}42 . \\
86\end{array}$ & 1 & $\begin{array}{l}14 . \\
29\end{array}$ & 0 & $\begin{array}{c}0.0 \\
0\end{array}$ & 7 & 1.44 & & \\
\hline \multicolumn{13}{|c|}{ What is your source of drinking water? } \\
\hline Bottled water & 116 & $\begin{array}{l}37 . \\
91\end{array}$ & 155 & $\begin{array}{l}50 . \\
65\end{array}$ & 25 & $\begin{array}{c}8.1 \\
7\end{array}$ & 10 & $\begin{array}{c}3.2 \\
7\end{array}$ & 306 & $\begin{array}{c}62.9 \\
6\end{array}$ & 9.2761 & $\begin{array}{c}0.158 \\
7\end{array}$ \\
\hline Well water & 9 & $\begin{array}{l}56 . \\
25\end{array}$ & 7 & $\begin{array}{l}43 . \\
75\end{array}$ & 0 & $\begin{array}{c}0.0 \\
0\end{array}$ & 0 & $\begin{array}{c}0.0 \\
0\end{array}$ & 16 & 3.29 & & \\
\hline Tap/Public water & 0 & $\begin{array}{c}0.0 \\
0\end{array}$ & 0 & $\begin{array}{c}0.0 \\
0\end{array}$ & 0 & $\begin{array}{c}0.0 \\
0\end{array}$ & 0 & $\begin{array}{c}0.0 \\
0\end{array}$ & 0 & 0.00 & & \\
\hline $\begin{array}{l}\text { Both tap/bottled/well } \\
\text { water }\end{array}$ & 74 & $\begin{array}{l}45 . \\
12 \\
\end{array}$ & 82 & $\begin{array}{l}50 . \\
00\end{array}$ & 5 & $\begin{array}{c}3.0 \\
5 \\
\end{array}$ & 3 & $\begin{array}{c}1.8 \\
3 \\
\end{array}$ & 164 & $\begin{array}{c}33.7 \\
4\end{array}$ & & \\
\hline \multicolumn{13}{|c|}{ What is the type of tea or fish do you take? } \\
\hline None & 8 & $\begin{array}{l}30 . \\
77\end{array}$ & 16 & $\begin{array}{l}61 . \\
54\end{array}$ & 2 & $\begin{array}{c}7.6 \\
9\end{array}$ & 0 & $\begin{array}{c}0.0 \\
0\end{array}$ & 26 & 5.35 & 19.2352 & $\begin{array}{c}0.377 \\
5\end{array}$ \\
\hline Tea & 6 & $\begin{array}{l}31 . \\
58\end{array}$ & 10 & $\begin{array}{l}52 . \\
63\end{array}$ & 2 & $\begin{array}{l}10 . \\
53\end{array}$ & 1 & $\begin{array}{c}5.2 \\
6\end{array}$ & 19 & 3.91 & & \\
\hline Coffee & 7 & $\begin{array}{c}50 . \\
00\end{array}$ & 6 & $\begin{array}{l}42 . \\
86\end{array}$ & 1 & $\begin{array}{c}7.1 \\
4\end{array}$ & 0 & $\begin{array}{c}0.0 \\
0\end{array}$ & 14 & 2.88 & & \\
\hline Both tea/coffee & 1 & $\begin{array}{c}6.6 \\
7\end{array}$ & 12 & $\begin{array}{c}80 . \\
00\end{array}$ & 1 & $\begin{array}{c}6.6 \\
7\end{array}$ & 1 & $\begin{array}{c}6.6 \\
7\end{array}$ & 15 & 3.09 & & \\
\hline Sea-fish (fresh) & 10 & $\begin{array}{l}34 . \\
48\end{array}$ & 17 & $\begin{array}{l}58 . \\
62\end{array}$ & 1 & $\begin{array}{c}3.4 \\
5\end{array}$ & 1 & $\begin{array}{c}3.4 \\
5\end{array}$ & 29 & 5.97 & & \\
\hline Dry-fish & 20 & $\begin{array}{l}38 . \\
46\end{array}$ & 28 & $\begin{array}{l}53 . \\
85\end{array}$ & 1 & $\begin{array}{c}1.9 \\
2\end{array}$ & 3 & $\begin{array}{c}5.7 \\
7\end{array}$ & 52 & $\begin{array}{c}10.7 \\
0\end{array}$ & & \\
\hline Others & 147 & $\begin{array}{l}44 . \\
41\end{array}$ & 155 & $\begin{array}{l}46 . \\
83\end{array}$ & 22 & $\begin{array}{c}6.6 \\
5\end{array}$ & 7 & $\begin{array}{c}2.1 \\
1\end{array}$ & 331 & $\begin{array}{c}68.1 \\
1\end{array}$ & & \\
\hline Total & 199 & $\begin{array}{l}40 . \\
95 \\
\end{array}$ & 244 & $\begin{array}{l}50 . \\
21 \\
\end{array}$ & 30 & $\begin{array}{c}6.1 \\
7 \\
\end{array}$ & 13 & $\begin{array}{c}2.6 \\
7 \\
\end{array}$ & 486 & $\begin{array}{c}100 . \\
00\end{array}$ & & \\
\hline
\end{tabular}

Subjects who visited the dentist in the past month were $59.47 \%$ and the most common reason for their visit was fluorosis $(40.33 \%)$. The subjects $(96.91 \%)$ started using toothpaste after 24 months and $60.70 \%$ of the subjects used pea sized amount of toothpaste for brushing. The subjects $(97.33 \%)$ rinsed and spat out after brushing routinely. Maximum
(98.77\%) number of the subjects used standard concentration of $\mathrm{Fl}$ paste for brushing. Fluoride supplements were not used by $64.40 \%$ of the subjects and fluoride gel/mouth rinse were used by $35.60 \%$ of the subjects. $99.38 \%$ of the children apply toothpaste by themselves while brushing (Table-6). 
Table-6: Access to dental services wise distribution of respondents

\begin{tabular}{|l|c|c|}
\hline Access to dental services & No of respondents & \% of respondents \\
\hline \multicolumn{3}{|c|}{ Did you visit the dentist in the last 12months? } \\
\hline Yes & 289 & 59.47 \\
\hline No What is the reason for the visit? \\
\hline \multicolumn{3}{|c|}{ 197 } \\
\hline Routine & 125 & 25.72 \\
\hline Caries & 58 & 11.93 \\
\hline Pain & 0 & 0.00 \\
\hline Fluorosis What age did you start using toothpaste? \\
\hline Stains & 15 & 40.33 \\
\hline \multicolumn{3}{|c|}{ Wh } \\
\hline Before 24 months & 471 & 3.09 \\
\hline After 24months & 107 & 96.91 \\
\hline
\end{tabular}

What is the amount of toothpaste used while brushing?

\begin{tabular}{|l|c|c|}
\hline Pea-sized & 295 & 60.70 \\
\hline Smear sized & 86 & 17.70 \\
\hline Full-brush head size & 105 & 21.60 \\
\hline
\end{tabular}

What do you do after brushing routinely?

\begin{tabular}{|l|c|c|}
\hline Swallowed paste & 13 & 2.67 \\
\hline Rinsed and spat out & 473 & 97.33 \\
\hline
\end{tabular}

What type of toothpaste did you use when you started brushing?

\begin{tabular}{|l|c|c|}
\hline Standard conc Fl paste & 480 & 98.77 \\
\hline Low conc Fl paste & 6 & 1.23 \\
\hline \multicolumn{2}{|c|}{ What type of fluoride supplements did you use in childhood? } \\
\hline Not used any & 313 & 64.40 \\
\hline Fluoride gel/mouth rinse & 173 & 35.60 \\
\hline Fluoride tablets & 0 & 0.00 \\
\hline
\end{tabular}

What is the frequency of tooth brushing in your childhood?

\begin{tabular}{|l|l|l|}
\hline Once/day or less & 111 & 22.84 \\
\hline Twice/day or more & 375 & 77.16 \\
\hline
\end{tabular}

Do you swallow toothpaste while brushing your teeth?

\begin{tabular}{|c|c|c|}
\hline Yes & 1 & 0.21 \\
\hline No & 485 & 99.79 \\
\hline \multicolumn{3}{|c|}{ Do you use fluoride supplements? } \\
\hline Yes & 162 & 33.33 \\
\hline No & 324 & 66.67 \\
\hline \multicolumn{3}{|c|}{ Who applies toothpaste to your toothbrush? } \\
\hline Child & 483 & 99.38 \\
\hline Parent & 3 & 0.62 \\
\hline Total & 486 & 100.00 \\
\hline
\end{tabular}

There was no statistically significant difference among categories of fluorosis according to the past dentist visit from the past 12 month, the reason associated with the dental visit, age of tooth brush initiation, amount of toothpaste used for brushing, type of toothpaste and fluoride supplement. When subject's frequency of tooth brushing in their childhood and use of fluoride supplements was compared statistically according to categories of enamel fluorosis, it was found to be statistically significant (Table-7). 
Eroje Alezi Braimoh Ifindon et al; Saudi J Oral Dent Res, Sep 2019; 4(9): 612-622

Table-7: Association between access to dental services and prevalence of enamel fluorosis

\begin{tabular}{|c|c|c|c|c|c|c|c|c|c|c|c|c|}
\hline Factors & $\begin{array}{l}\text { Nor } \\
\text { mal }\end{array}$ & $\%$ & $\begin{array}{c}\text { Questiona } \\
\text { ble }\end{array}$ & $\%$ & $\begin{array}{l}\text { Very } \\
\text { mild }\end{array}$ & $\%$ & $\begin{array}{c}\text { Mil } \\
\mathrm{d}\end{array}$ & $\%$ & $\begin{array}{c}\text { Tot } \\
\text { al }\end{array}$ & $\%$ & $\begin{array}{c}\text { Chi- } \\
\text { square }\end{array}$ & $\begin{array}{c}\mathrm{p}- \\
\text { value }\end{array}$ \\
\hline \multicolumn{13}{|c|}{ Did you visit the dentist for the last 12 months? } \\
\hline Yes & 119 & 41.18 & 137 & 47.40 & 23 & 7.96 & 10 & 3.46 & 289 & 59.47 & 6.4498 & 0.0917 \\
\hline No & 80 & 40.61 & 107 & 54.31 & 7 & 3.55 & 3 & 1.52 & 197 & 40.53 & & \\
\hline \multicolumn{13}{|c|}{ What is the reason for the visit? } \\
\hline Routine & 56 & 44.80 & 52 & 41.60 & 12 & 9.60 & 5 & 4.00 & 125 & 25.72 & 15.0044 & 0.0909 \\
\hline Caries & 22 & 37.93 & 32 & 55.17 & 1 & 1.72 & 3 & 5.17 & 58 & 11.93 & & \\
\hline Pain & 0 & 0.00 & 0 & 0.00 & 0 & 0.00 & 0 & 0.00 & 0 & 0.00 & & \\
\hline Fluorosis & 80 & 40.82 & 106 & 54.08 & 7 & 3.57 & 3 & 1.53 & 196 & 40.33 & & \\
\hline stains & 41 & 38.32 & 54 & 50.47 & 10 & 9.35 & 2 & 1.87 & 107 & 22.02 & & \\
\hline \multicolumn{13}{|c|}{ What age did you start using toothpaste? } \\
\hline Before 24 months & 6 & 40.00 & 7 & 46.67 & 2 & $\begin{array}{c}13.3 \\
3\end{array}$ & 0 & 0.00 & 15 & 3.09 & 1.7416 & 0.6277 \\
\hline After 24 months & 193 & 40.98 & 237 & 50.32 & 28 & 5.94 & 13 & 2.76 & 471 & 96.91 & & \\
\hline \multicolumn{13}{|c|}{ What is the amount of toothpaste used while brushing? } \\
\hline Pea-sized & 114 & 38.64 & 149 & 50.51 & 22 & 7.46 & 10 & 3.39 & 295 & 60.70 & 5.1657 & 0.5228 \\
\hline Smear sized & 41 & 47.67 & 41 & 47.67 & 3 & 3.49 & 1 & 1.16 & 86 & 17.70 & & \\
\hline Full-brush head size & 44 & 41.90 & 54 & 51.43 & 5 & 4.76 & 2 & 1.90 & 105 & 21.60 & & \\
\hline \multicolumn{13}{|c|}{ What do you do after brushing routinely? } \\
\hline Swallowed paste & 6 & 46.15 & 5 & 38.46 & 1 & 7.69 & 1 & 7.69 & 13 & 2.67 & 1.7625 & 0.6231 \\
\hline Rinsed and spat out & 193 & 40.80 & 239 & 50.53 & 29 & 6.13 & 12 & 2.54 & 473 & 97.33 & & \\
\hline \multicolumn{13}{|c|}{ What type of toothpaste did you use when you started brushing? } \\
\hline $\begin{array}{lll}\begin{array}{l}\text { Standard } \\
\text { paste }\end{array} & \text { conc } & \mathrm{Fl} \\
\end{array}$ & 198 & 41.25 & 240 & 50.00 & 30 & 6.25 & 12 & 2.50 & 480 & 98.77 & 6.0236 & 0.1105 \\
\hline low conc $\mathrm{Fl}$ paste & 1 & 16.67 & 4 & 66.67 & 0 & 0.00 & 1 & $\begin{array}{c}16.6 \\
7\end{array}$ & 6 & 1.23 & & \\
\hline \multicolumn{13}{|c|}{ What type of fluoride supplements did you use in childhood? } \\
\hline Not used any & 126 & 40.26 & 165 & 52.72 & 14 & 4.47 & 8 & 2.56 & 313 & 64.40 & 5.3690 & 0.1467 \\
\hline $\begin{array}{l}\text { Fluoride gel/mouth } \\
\text { rinse }\end{array}$ & 73 & 42.20 & 79 & 45.66 & 16 & 9.25 & 5 & 2.89 & 173 & 35.60 & & \\
\hline Fluoride tablets & 0 & 0.00 & 0 & 0.00 & 0 & 0.00 & 0 & 0.00 & 0 & 0.00 & & \\
\hline \multicolumn{13}{|c|}{ What is the frequency of tooth brushing in your childhood? } \\
\hline Once/day or less & 55 & 49.55 & 50 & 45.05 & 2 & 1.80 & 4 & 3.60 & 111 & 22.84 & 8.2798 & $\begin{array}{c}0.0406 \\
*\end{array}$ \\
\hline Twice/day or more & 144 & 38.40 & 194 & 51.73 & 28 & 7.47 & 9 & 2.40 & 375 & 77.16 & & \\
\hline \multicolumn{13}{|c|}{ Do you swallow toothpaste while brushing your teeth? } \\
\hline Yes & 0 & 0.00 & 1 & $\begin{array}{c}100.0 \\
0\end{array}$ & 0 & 0.00 & 0 & 0.00 & 1 & 0.21 & 0.9938 & 0.8027 \\
\hline No & 199 & 41.03 & 243 & 50.10 & 30 & 6.19 & 13 & 2.68 & 485 & 99.79 & & \\
\hline \multicolumn{13}{|c|}{ Do you use fluoride supplements? } \\
\hline Yes & 70 & 43.21 & 71 & 43.83 & 16 & 9.88 & 5 & 3.09 & 162 & 33.33 & 7.8271 & $\begin{array}{c}0.0497 \\
*\end{array}$ \\
\hline No & 129 & 39.81 & 173 & 53.40 & 14 & 4.32 & 8 & 2.47 & 324 & 66.67 & & \\
\hline \multicolumn{13}{|c|}{ Who applies toothpaste to your toothbrush? } \\
\hline Child & 199 & 41.20 & 241 & 49.90 & 30 & 6.21 & 13 & 2.69 & 483 & 99.38 & 2.9939 & 0.3926 \\
\hline Parent & 0 & 0.00 & 3 & $\begin{array}{c}100.0 \\
0\end{array}$ & 0 & 0.00 & 0 & 0.00 & 3 & 0.62 & & \\
\hline Total & 199 & 40.95 & 244 & 50.21 & 30 & 6.17 & 13 & 2.67 & 486 & $\begin{array}{c}100.0 \\
0\end{array}$ & & \\
\hline
\end{tabular}

\section{DISCUSSION}

In spite of the considerable studies in the western context on fluorosis, risk factors associated with dental fluorosis are still not clear especially in Middle East. Therefore, the present study was conducted in Abha-Khamis region using a probability purposive sampling methodology in the selected female schools so that statistical relationship between dental fluorosis and the associated risk factors can be established.
In the current research, prevalence of dental fluorosis was found in 287subjects among total screened girls $(n=486)$. Hence, the prevalence of dental fluorosis was $59.05 \%$. Questionable fluorosis got highest proportion which was $50.21 \%$ followed by very mild fluorosis (6.17\%). Soban Qadir Khan et al., [12] in his study reported dental fluorosis in $28 \%$ of girls and mild fluorosis was the most prevalent type. Different pattern of fluorosis had been observed previously in the 
studies conducted in different regions of Saudi Arabia. In those studies, mild level of fluorosis was observed in most of the children who were affected from fluorosis. Proportion of mild fluorosis was $21.8 \%$ followed by moderate level which was 5.3\% [13]. In Riyadh, Saudi Arabia, 5-12-year-old children were examined for oral health survey. Fluorosis was found in only $14 \%$ of total cases and all had mild level of fluorosis [14]. Moderate and severe level of fluorosis was reported from Western Sahara. A study conducted among refugee children observed that only $4 \%$ of $11-13$-year-old children were free from fluorosis and almost $30 \%$ of them were affected from moderate fluorosis and prevalence of severe fluorosis was among $27 \%$ of total screened children [15]. This difference in categories of fluorosis indicates that more epidemiological studies are needed to record the prevalence and severity of the disease due to its significant variability in different regions.

We found in this present study that there is statistical significance association between age in years, type of school and location-type school and the prevalence of dental fluorosis. The prevalence of dental fluorosis and its severity was higher in the urban area compared to that in the rural area, and in younger children compared to older ones. Rarely previous studies have not noted any urban-rural differences in the prevalence of dental fluorosis. We believe that the urban-rural differences in our study area may be related in part to the increased use of pipe and bottled water for drinking purposes in the urban areas; we have presented data to substantiate that the intake of pipe/bottled water was associated with a higher prevalence of fluorosis compared to consumption of well water. Similar results were revealed by P. Gopalakrishnan et al., [1]

The prevalence of dental fluorosis was also greater in the younger age groups. One possible explanation by P. Gopalakrishnan et al., [1] for this intriguing observation is that younger children drink more pipe/bottled water compared to older children. This trend of a higher prevalence of fluorosis in younger age groups can be further examined by studying the prevalence of dental fluorosis in younger children in the study area. A study of the prevalence of skeletal fluorosis and non-skeletal manifestations in the area (with the cooperation of orthopaedic experts and gastroenterologists) may also shed light on this observation.

The socio-economic status of the parents had no influence on the prevalence of dental fluorosis in our study area. This is similar to the results revealed by $\mathrm{P}$. Gopalakrishnan et al., [1] but contrary to a prior report that described a higher prevalence of fluorosis in children belonging to a high socio-economic status. Furthermore, different types of tea drinking and sea- or dry-fish consumption were not associated with the prevalence of dental fluorosis which is in accordance with the results found by P. Gopalakrishnan et al., [1]
In the present study, very mild and mild fluorosis was reported most in subjects whose mothers were illiterate. This could be related to the fact that the higher amounts of dentifrice dispensed to these children have been associated with lower socioeconomic status and parental education level. Although children from higher socioeconomic status tend to use children's toothpaste, instead of the family dentifrice, the amount of dentifrice used can strongly increase the risk of exposure to higher doses of Fluoride, regardless of the type of dentifrice. Maltz and Silva [16] found that children who attended private schools had a higher prevalence of dental fluorosis, but found no direct association between either family income or parental schooling. Similarly, other authors have found no association between dental fluorosis and socioeconomic indicators. This finding is also consistent with this present study.

Surprisingly in the present study, the prevalence of dental fluorosis is high among children who used bottled water as compared to those who use well water for drinking purposes which is similar to the study done by Hazza A Alhobeira [17]. P. Gopalakrishnan et al., [1] in his study reported high prevalence of dental fluorosis among children who used pipe water for drinking purposes. The reason for this may be that the fluoride content of pipe water is higher compared to that of other water sources. It is likely that domestic wells and those bored by the water authority vary both in their location and depth, and consequently in the fluoride content of their water. It has been previously demonstrated that even within a small community, different wells have widely varying water fluoride content. This is related in part to divergent hydro-geological conditions; the proportion of rocks with readily leachable fluoride can vary substantially within a given geographical belt.

Use of Fluoride toothpaste has been identified as a potential risk factor for dental fluorosis, particularly because an inverse relationship has been observed between age and mean ingestion of toothpaste [18]. Both the frequency of tooth brushing $(\mathrm{p}=0.0406)$ and the use of fluoride supplements $(\mathrm{p}=0.0497)$ and the age $(\mathrm{p}=0.0489)$ at which tooth brushing began were found to be associated with dental fluorosis in the present study. It has been reported that an early age of tooth brushing initiation is associated with higher fluorosis. The authors [18] of the above-mentioned finding presumed this was connected to the lack of supervision in young children to advise them to spit out toothpaste rather than swallow it. Since most commercial brands of toothpaste are fluoridated, they serve as important sources of Fluoride, but present substantial risk associated with ingestion by children. It has been shown that not all ingested Fluoride from toothpaste will be absorbed, and may not be absorbed systemically $[19,20]$. The finding that children who initiated tooth brushing at an early age had a higher prevalence of dental fluorosis may suggest that they 
swallowed a quantity of fluoridated toothpaste great enough to have had a negative systemic impact.

An association between a higher frequency of tooth brushing and fluorosis was detected in this study, in agreement with other studies [21, 22]. Although, the frequency of tooth brushing was not precise, it was used as a proxy measurement for the amount of Fluoride toothpaste being ingested, insofar as higher frequencies of tooth brushing are expected to increase the risk of Fluoride intake ${ }^{19}$. Although other proxy measurements have been suggested, including those of collecting information on whether the child liked, swallowed or ate dentifrices, this study only assessed the current tooth brushing practices of the sample [23]. It is possible that these children engaged in more frequent tooth brushing ever since they began brushing their teeth. This possibility is supported by Pendrys et al., [24] who found that $34 \%$ of the cases of Dental fluorosis could be explained by initiation of tooth brushing more often than once a day and brushing with Fluoride toothpaste at an age under 2 years of age. On the other hand, Wong et al., [23] performed a meta-analysis of four crosssectional surveys and found no significant association between frequency of tooth brushing and dental fluorosis. The findings of this study suggest that advising parents on the proper use of Fluoride dentifrices by their children and urging them to supervise their children during tooth brushing are recommendations widely accepted by dentists, and add to the benefits of reducing the risk of dental fluorosis. Each parent should be well informed about the many benefits and little damage associated with brushing their children's teeth with Fluoride toothpaste.

\section{Limitations of this Study}

This is a cross-sectional study and therefore, limits the extent to which causal inferences could be made. The major risk factor in the development of dental fluorosis is drinking water. Fluorosis develops in an individual during the time of calcification of teeth, which takes place from early infancy. The fluoride content of the water which was consumed during that period is of critical importance, but interestingly the fluoride content remains within optimal level. It is presumed in this study that the fluoride content of water in each area has not changed over the last 15 years. Another limitation was the possibility of recall bias, since mothers were requested to answer questions regarding their children's exposure to Fluoride during early childhood.

\section{CONCLUSIONS}

Dental fluorosis is a major public health issue in the Southern sub-population (Abha and Khamis) of Saudi Arabia. In this present study borderline public health significance was seen with a CFI of 0.4 . The prevalence of dental fluorosis in this sample of students was high, but the fluorosis was of low severity with no single case of moderate and severe form of fluorosis reported. The principal factor associated with the presence of dental fluorosis was the frequency of tooth brushing and the use of fluoride supplements with a statistical significance level $(\mathrm{p}=0.04060$ and $(\mathrm{p}=0.0497)$ respectively in this tested model. Active steps/strategies must be taken to educate the community on the correct frequency of tooth brushing during childhood development and the medical practitioners on the correct prescription of fluorides supplements during the stages of tooth development.

\section{ACKNOWLEDGEMENTS}

The authors wish to thank the officials of the Ministry of Education for permitting the conduct of this survey at selected schools; the school teachers and staff for their collaboration. Special thanks also to Dr. Asiri HOD and staff of department of Chemistry, King Khalid University, Abha who provide the expertise in the analysis of fluoride content in the various sample of water.

\section{Declaration of conflict of Interest and Funding}

The authors clarify that there is no conflict of interest with respect to the authorship and or publication of this manuscript. The author received no financial support or grant from grant awarding body for the research and or authorship of this manuscript.

\section{Authorship}

The authors Drs, Alezi Braimoh Ifindon Eroje, Shreyas Tikare, Shaeesta Khaleelahmed B, Batool Mohammed Al-wadai, Shahad Moshhen Al-beshi were involved in the concept and design of this study, collection, analysis and interpretation of data. Literature search and the final drafting of the manuscript were done by Dr. Eroje ABI. Revising and editing of the manuscript was done by Dr. Shaeesta Khaleelahmed B and Dr.Samuel Ebele Udeabor. All the authors approved the submission of the manuscript to the Nigerian Journal of Dental Research. The authors take responsibility for the integrity of this work as a whole from inception to publication and give the rights to the corresponding author, Dr Eroje ABI to make necessary changes as per the request of the journal, do the rest of the correspondence on our behalf and he will act as the guarantor for the manuscript.

\section{REFERENCES}

1. Gopalakrishnan, P., Vasan, R. S., Sarma, P. S., Ravindran Nair, K. S., \& Thankappan, K. R. (1999). Prevalence of dental fluorosis and associated risk factors in Allappuzha district, Kerala. National Medical Journal of India, 12, 99102.

2. Grimaldo, M., Borjaaburto, V. H., Ramirez, A. L., Ponce, M., Rosas, M., \& Diazbarriga, F. (1995). Endemic fluorosis in San-Luis-Potosi, Mexico. 1. Identification of risk-factors associated with human 
Eroje Alezi Braimoh Ifindon et al; Saudi J Oral Dent Res, Sep 2019; 4(9): 612-622

exposure to fluoride. Environmental

Research, 68(1), 25-30.

3. Akpata, E. S. (2001). Occurrence and management of dental fluorosis. International dental journal, 51(5), 325-333.

4. Kotecha, P. V., Patel, S. V., Bhalani, K. D., Shah, D., Shah, V. S., \& Mehta, K. G. (2012). Prevalence of dental fluorosis \& dental caries in association with high levels of drinking water fluoride content in a district of Gujarat, India. The Indian journal of medical research, 135(6), 873-877.

5. Siddiqui, A. A., Al Hobeira, H., Mirza, A. J., Alshammari, A. K., Alshammari, B. A., \& Alsalwah, N. H. (2017). Dental Fluorosis in Saudi Arabia: A review of current literature. Annals of International Medical and Dental Research,3(3), 44-49.

6. Pontigo-Loyola, A. P., Islas-Márquez, A., LoyolaRodríguez, J. P., Maupome, G., Marquez-Corona, M. L., \& Medina-Solis, C. E. (2008). Dental Fluorosis in 12-and 15-Year-Olds at High Altitudes in Above-Optimal Fluoridated Communities in Mexico. Journal of public health dentistry, 68(3), 163-166.

7. Nakornchai, S., Hopattaraput, P., \& Vichayanrat, T. (2016). Prevalence, Severity And Factors Associated With Dental Fluorosis Among Children Aged 8-10 Years In Bangkok, Thailand. The Southeast Asian journal of tropical medicine and public health, 47(5), 1105-1111.

8. Rigo, L., Caldas Junior, A. D. F., \& Souza, E. H. A. D. (2010). Factors associated with dental fluorosis. Revista Odonto Ciência, 25(1), 8-14.

9. Abdul Salam, A. (2013). Population and household census, Kingdom of Saudi Arabia 2010: facts and figures. International Journal Humanities and Social Science, 3(16).

10. Mackey, S. (2002). The Saudis: Inside the desert kingdom. WW Norton \& Company.

11. World Health Organization. (2013). Oral health surveys: basic methods 5th edition. World Health Organization.

12. Khan, S. Q., Moheet, I. A., Farooq, I., Farooqi, F. A., ArRejaie, A. S., Al Abbad, M. H. A., \& Khabeer, A. (2015). Prevalence of dental fluorosis in school going children of Dammam, Saudi Arabia. Journal of Dental and Allied Sciences, 4(2), 69-72.

13. AlDosari, A. M., Akpata, E. S., \& Khan, N. (2010). Associations among dental caries experience, fluorosis, and fluoride exposure from drinking water sources in Saudi Arabia. Journal of public health dentistry, 70(3), 220-226.
14. Al-Banyan, R. A., Echeverri, E. A., Narendran, S., \& Keene, H. J. (2000). Oral health survey of 5-12year-old children of National Guard employees in Riyadh, Saudi Arabia. International journal of paediatric dentistry, 10(1), 39-45.

15. Almerich-Silla, J. M., Montiel-Company, J. M., \& Ruiz-Miravet, A. (2008). Caries and dental fluorosis in a western Saharan population of refugee children. European journal of oral sciences, 116(6), 512-517.

16. Maltz, M., \& Silva, B. B. (2001). Relationship between caries, gingivitis and fluorosis and the socioeconomic status among school children. Revista de saude publica, 35(2), 170-176.

17. Alhobeira, H. A., Siddiqui, A. A., \& Mian, R. I. (2015). Prevalence and severity of dental fluorosis in Hail, Saudi Arabia. Journal of International Oral Health, 7(12), 1-4.

18. Buzalaf, M. A. R. (Ed.). (2011). Fluoride and the oral environment (Vol. 22). Karger Medical and Scientific Publishers.

19. Martins, C. C., Oliveira, M. J., Pordeus, I. A., Cury, J. A., \& Paiva, S. M. (2011). Association between socioeconomic factors and the choice of dentifrice and fluoride intake by children. International Journal of Environmental Research and Public Health, 8(11), 4284-4299.

20. Falcão, A., Tenuta, L. M. A., \& Cury, J. A. (2013). Fluoride gastrointestinal absorption from $\mathrm{Na} 2 \mathrm{FPO} 3 / \mathrm{CaCO} 3$-and $\quad \mathrm{NaF} / \mathrm{SiO} 2$-based toothpastes. Caries research, 47(3), 226-233.

21. Alicia Vallejos-Sánchez, A., Eduardo MedinaSolís, C., Fernando Casanova-Rosado, J., Maupomé, G., Minaya-Sánchez, M., \& PérezOlivares, S. (2006). Dental fluorosis in cohorts born before, during, and after the national salt fluoridation program in a community in Mexico. Acta Odontologica Scandinavica, 64(4), 209-213.

22. Pendrys, D. G., Katz, R. V., \& Morse, D. E. (1994). Risk factors for enamel fluorosis in a fluoridated population. American journal of epidemiology, 140(5), 461-471.

23. Wong, M. C., Glenny, A. M., Tsang, B. W., Lo, E. C., Worthington, H. V., \& Marinho, V. C. (2010). Topical fluoride as a cause of dental fluorosis in children. Cochrane Database of Systematic Reviews, (1):CD007693.

24. Pendrys, D. G., Haugejorden, O., Baårdsen, A., Wang, N. J., \& Gustavsen, F. (2010). The risk of enamel fluorosis and caries among Norwegian children: implications for Norway and the United States. The Journal of the American Dental Association, 141(4), 401-414. 